Unabhängiges Landeszentrum für Datenschutz Schleswig-Holstein (ULD)

\title{
EuroPriSe 2.0 - Fortführung des Europäischen Datenschutzgütesiegels
}

Das EuroPriSe-Gütesiegel ${ }^{1}$, die Zertifizierung von IT-Produkten und IT-basierten Dienstleistungen auf ihre Datenschutzkonformität nach europäischem Recht, geht zum 01.01.2014 vom Unabhängigen Landeszentrum für Datenschutz Schleswig-Holstein (ULD) auf die EuroPriSe GmbH über.

Die EuroPriSe GmbH wird die Zertifizierung fortführen und im Einklang mit einem unabhängigen Expertengremium weiterentwickeln. „Ich freue mich, dass wir EuroPriSe nun mit der Gründung der EuroPriSe GmbH die nötigen Wachstumsmöglichkeiten geben können, die uns im Rahmen einer behördlichen Struktur und deren karger Ausstattung verwehrt waren“, erläutert Thilo Weichert, Leiter des ULD und Landesbeauftragter für Datenschutz Schleswig-Holstein, den Grund für die neue Trägerstruktur.

Die EuroPriSe GmbH hat sich gegenüber dem ULD vertraglich verpflichtet, den bewährten europäischen Datenschutzkriterienkatalog fortzuführen. Das ULD wird als Mitglied des Expertengremiums EuroPriSe weiter begleiten. „Zwar ändert sich die Eigentümerstruktur; Unabhängigkeit und Verfahren des europäischen Gütesiegels bleiben jedoch bestehen“, erklärt Marcus Belke, Geschäftsführer der EuroPriSe GmbH. Alle akkreditierten Experten werden von der neuen Eigentümerin anerkannt, sämtliche Zertifizierungsverfahren können fortgeführt werden.

Mit der neuen Struktur besteht die Möglichkeit, die erlangten Erfahrungen auf die Zertifizierung von Verfahren, Verarbeitungen im Auftrag, Konzepten, Personen, Schulungen oder Webseiten zu übertragen und gemäß den Vorgaben der EU-Datenschutzgesetzgebung und den Festlegungen der Art. 29-Datenschutzgruppe weiterzuentwickeln. Vorbild hierfür wird das bewährte EuroPriSe-Modell sein mit der Schulung und Akkreditierung von Prüfern, der Prüfung durch EuroPriSe-Experten entlang objektiver Schemata und der abschließenden Validierung und Zertifizierung. Gleichzeitig wird die EuroPriSe GmbH den Herstellern und Experten räumlich entgegenkommen, zum Beispiel durch Schulungsangebote in weiteren Mitgliedsstaaten der EU und lokalen Dependancen. „Ein solcher Ausbau unter Beibebehaltung der bewährten EuroPriSe-Ansprüche wäre unter dem Dach einer Behörde nicht möglich gewesen. Die internationalen EuroPriSe-Experten erhalten so die Möglichkeit, an einem brei-

1 https://www.datenschutzzentrum.de/europrise/20131114-factsheet.pdf PDF ten Spektrum von Datenschutzzertifizierungen mitzuwirken und ihr Geschäftsfeld auszubauen. Den Firmenkunden wird eine breitere Auswahl an EuroPriSe-Zertifizierungen angeboten“, erläutert Marcus Belke die Expansionspläne von EuroPriSe.

Gründungsgesellschafter der EuroPriSe GmbH ist die 2B Advice-Gruppe, eine internationale Beratungsgruppe für Datenschutz, die mit ihren Beratungsgesellschaften in mehreren Ländern Europas und in den USA vertreten ist. „2B Advice hat unter weiteren Bewerbern das überzeugendste Konzept für die unabhängige Führung der EuroPriSe GmbH vorgelegt“, sagt Thilo Weichert. Weitere Informationen zu diesem Konzept können dem beigefügten Factsheet entnommen werden.

Im Interesse einer langfristigen Qualitätssicherung des EuroPriSe-Siegels hat sich die EuroPriSe GmbH verpflichtet,

1. die Weiterentwicklung des EuroPriSe-Projekts durch die Bildung eines unabhängigen Expertengremiums zu begleiten und eng mit der Art. 29-Datenschutzgruppe zusammenzuarbeiten,

2. die Transparenz der Verfahrensabläufe und die Veröffentlichung der EuroPriSe-Kriterien beizubehalten,

3. die Zweistufigkeit des Verfahrens, die europäische Ausrichtung und die Orientierung am hohen Datenschutzstandard, wie er von der Art. 29-Datenschutzgruppe und den nationalen Aufsichtsbehörden definiert wird, zu bewahren,

4. neue Schemata und Änderungen an bestehenden Schemata sowie Zertifizierungsanforderungen dem ULD und Aufsichtsbehörden in weiteren EU-Ländern zur Kommentierung vorzulegen und deren Ergebnisse zu berücksichtigen,

5. die bisherigen Akkreditierungen der Gutachter zu akzeptieren,

6. die Ausbildung der Gutachter an mehreren Standorten international fortzuführen und auszubauen,

7. das Register der verliehenen Gütesiegel zu übernehmen und weiterzuführen,

8. die Prüfung eingereichter Gutachten zeitnah und in hoher Qualität durchzuführen,

9. die Kosten für die Akkreditierung zu überarbeiten und den nationalen Gegebenheiten anzupassen sowie

10. die Kosten der Zertifizierungen weiterhin erfolgsunabhängig zu gestalten. 